\title{
Efeitos de um programa de intervenção de atividade física, educação e promoção de saúde com idosos hipertensos usuários do Sistema Único de Saúde
}

\author{
Effects of an intervention program on physical activity, education and health promotion \\ with hypertensive elderly users of the Unified Health System
}

Efectos de un programa de intervención de actividad física, educación y promoción de la salud en usuarios mayores hipertensos del Sistema Único de Salud

Gizele Santiago de Moura Silva ${ }^{1}$, Paulo Roberto Cavalcanti Carvalho ${ }^{1 *}$, José Cristiano Faustino dos Santos ${ }^{1}$, Edigleide Maria Figueiroa Barreto ${ }^{1}$, Esdras Henrique Ragel de Meloㄹ, Juan Carlos Freire ${ }^{1}$, Gustavo Willames Pimentel Barros².

\section{RESUMO}

Objetivo: Avaliar o efeito de um programa de intervenção de atividade física, educação e promoção de saúde com idosos hipertensos do Sistema Único de Saúde. Métodos: Estudo transversal com abordagem quantitativa, realizada com idosos hipertensos cadastrados nas unidades de saúde da família, com idade $\geq 60$ anos. Foram aplicados questionários para nível socioeconômico, nível de atividade física, aferição da pressão arterial e avaliação antropométrica, foram passadas orientações e um manual ilustrativo sobre a realização da prática de atividade física e hábitos saudáveis. Após dois meses foram reavaliados. Resultados: Pré e Pós intervenção respectivamente nível de atividade física ativo $7 \%$ e $11 \%$, irregularmente ativo A $8 \%$ e 10\%, irregularmente ativo B 7\% e $8 \%$ e sedentário $27 \%$ e $20 \%$, PAS 137, $14 \%$ e $126,73 \%$, PAD 83,06 e 80,27 , peso $71,99 \%$ e $71,27 \%$, IMC $28,40 \%$ e $28,16 \%$, CP $36,16 \%$ e $35,71 \%$ e CABS $98,35 \%$ e $97,59 \%$. Conclusão: Houve melhora na pressão arterial, nos níveis de atividade física e em todas as medidas antropométricas pós intervenção, mas o risco cardiovascular permaneceu alto na maioria dos idosos. Assim, a intervenção realizada melhorou os níveis de atividade física, na pressão arterial e entre os aspectos antropométricos, mostrando uma melhor conscientização sobre hábitos saudáveis e estilo de vida.

Palavras-chave: Idosos, Hipertensão, Atividade física, Medidas, Aconselhamento.

\begin{abstract}
Objective: To evaluate the effect of an intervention program for physical activity, education and health promotion with hypertensive elderly people in the Unified Health System. Methods: Cross-sectional study with a quantitative approach, carried out with hypertensive elderly people registered in family health units, aged $\geq 60$ years. Questionnaires were applied for socioeconomic level, level of physical activity, blood pressure measurement and anthropometric assessment, guidelines and an illustrative manual on the practice of physical activity and healthy habits were given. After two months, they were reevaluated. Results: Pre and Post intervention respectively level of active physical activity $7 \%$ and $11 \%$, irregularly active $\mathrm{A} 8 \%$ and $10 \%$, irregularly active $\mathrm{B} 7 \%$ and $8 \%$ and sedentary $27 \%$ and $20 \%$, PAS $137.14 \%$ and 126 , $73 \%$, PAD 83.06 and 80.27 , weight $71.99 \%$ and $71.27 \%$, BMI $28.40 \%$ and $28.16 \%$, CP $36.16 \%$ and $35.71 \%$ and CABS $98.35 \%$ and $97.59 \%$. Conclusion: There was an improvement in blood pressure, levels of physical activity and in all anthropometric measures after the intervention, but the cardiovascular risk remained high in the majority of the elderly. Thus, the intervention performed improved levels of physical activity, blood pressure and among anthropometric aspects, showing a better awareness of healthy habits and lifestyle.
\end{abstract}

Keywords: Elderly, Hypertension, Physical activity, Measures, Counseling.

1 Universidade Federal de Pernambuco (UFPE), Recife - PE. *E-mail: paulo.robertocarvalho@ufpe.br

2 Universidade Federal de Paraíba (UFPB), João Pessoa - PB.

SUBMETIDO EM: 3/2021

ACEITO EM: 4/2021

PUBLICADO EM: 4/2021 


\section{RESUMEN}

Objetivo: Evaluar el efecto de un programa de intervención para la actividad física, educación y promoción de la salud en ancianos hipertensos del Sistema Único de Salud. Métodos: Estudio transversal con abordaje cuantitativo, realizado con ancianos hipertensos registrados en unidades de salud de la familia, edad $\geq 60$ años. Se aplicaron cuestionarios por nivel socioeconómico, nivel de actividad física, medición de la presión arterial y valoración antropométrica, se entregaron directrices y un manual ilustrativo sobre la práctica de actividad física y hábitos saludables. Después de dos meses, fueron reevaluados. Resultados: Pre y Post intervención respectivamente nivel de actividad física activa $7 \%$ y $11 \%$, irregularmente activo $\mathrm{A}$ $8 \%$ y $10 \%$, irregularmente activo B $7 \%$ y $8 \%$ y sedentario $27 \%$ y $20 \%$, PAS $137,14 \%$ y $126,73 \%$, PAD 83.06 y 80.27 , peso $71.99 \%$ y $71.27 \%$, IMC $28.40 \%$ y $28.16 \%$, CP $36.16 \%$ y $35.71 \%$ y CABS $98.35 \%$ y $97.59 \%$. Conclusión: Hubo una mejoría en la presión arterial, los niveles de actividad física y en todas las medidas antropométricas después de la intervención, pero el riesgo cardiovascular se mantuvo alto en la mayoría de los ancianos. Así, la intervención realizó una mejora en los niveles de actividad física, tensión arterial y entre aspectos antropométricos, mostrando una mejor conciencia de hábitos saludables y estilo la vida.

Palabras clave: Anciano, Hipertensión, Actividad física, Medidas, Asesoramiento.

\section{INTRODUÇÃO}

Á medida que o envelhecimento da população avança e o número de óbitos precoces diminui, aumenta a prevalência de doenças como a hipertensão e diabetes. Hoje é comprovando que a hipertensão é considerada uma das principais doenças cardiovasculares, apresentando assim um maior risco para o idoso como o do infarto agudo do miocárdio, coronoariopatias e acidente vascular cerebral atingindo assim 70\% desta população (MIRANDA GMD, et al., 2016; HERROD PJJ, et al., 2018; NASCHITZ JE, 2018).

A HA é uma condição clínica multifatorial caracterizada pelo aumento dos níveis pressóricos diagnosticada clinicamente nos indivíduos que apresentam níveis iguais ou superiores à $140 \mathrm{mmHg}$ na pressão arterial sistólica (PAS) e/ou níveis iguais ou superiores à $90 \mathrm{mmHg}$ na pressão arterial diastólica $(P A D)$. Esta é uma das doenças crônicas não transmissíveis mais prevalentes no mundo, sendo uma das causas de maior óbito, aumento do número de mortes prematuras e perda de qualidade de vida, principalmente em idosos (OMS, 2016; CASTRO L, et al., 2019)

Os custos para o controle e tratamento da HA no mundo foram de US $\$ 46,4$ bilhões em 2014, com previsão de aumentos de seis vezes desse valor até 2030. Devido a esses gastos se faz necessário e é importante o incentivo de políticas públicas para o uso de estratégias não farmacológicas para o combate da HA (MOZAFFARIAN D, et al., 2015; DAMMERO DR, et al., 2019).

É observado que o tratamento não farmacológico dessa condição clínica está associado a fatores relacionados às mudanças nos hábitos de vida, como a reeducação alimentar e inserção da prática regular do exercício físico (RAMOS RF, et al., 2018; OLIVEIRA VS, et al., 2017).

Dentre as terapias não farmacológicas para o controle da HA, a atividade física e a mudança de comportamento que têm se destacado, por se tratar de duas estratégias acessíveis e de baixo risco, sendo recomendadas por serem ambas aliadas no tratamento da hipertensão juntamente com hábitos alimentares saudáveis (FARIAS NO, et al., 2021).

A diminuição do peso corporal pode promover até $10 \mathrm{mmHg}$ a menos nos níveis pressóricos, conforme vai reduzindo o peso, então havendo o aumento da prática de atividade física, redução do estresse e do consumo de sal, dentre outras medidas favoráveis, pode ser possível a retirada do tratamento medicamentoso com manutenção dos níveis pressóricos dentro dos limites considerados normais (BRASIL, 2017).

Os programas de exercícios físicos representam uma importante estratégia na prevenção e tratamento, agindo diretamente na hipertensão, gerando assim um decréscimo temporário na tensão, mesmo após uma 
única sessão de exercício físico chamado de hipotensão pós-exercício, cujo fatores como duração, tipo e intensidade do exercício associados a níveis pressóricos de repouso podem influenciar a diminuição da pressão arterial (FERREIRA RC, et al., 2017; LEANDRO MPG, et al., 2019).

Entre as estratégias para melhorar os índices de saúde na atenção básica, o aconselhamento e programas de intervenção têm se tornado uma importante chave para se obter resultados positivos. De acordo com a Política Nacional de Promoção da Saúde (PNPS) de 2010, as orientações sobre hábitos adequados do estilo de vida são necessárias, considerando isso como um processo educativo que todos os profissionais de saúde devem realizar para instruir os pacientes a manterem uma vida mais saudável, permitindo que eles tenham maior autonomia e conhecimento sobre seu processo de saúde. Mas ainda assim, esse tipo de prática ainda não tem sido priorizado por grande parte dos profissionais e pelos serviços de saúde na atenção básica (HALL JE, et al., 2015; PORTES LA, et al., 2018).

Desta forma a presente pesquisa teve como objetivo avaliar o efeito de um programa de intervenção de atividade física, educação e promoção de saúde com idosos hipertensos usuários do Sistema Único de Saúde.

\section{MÉTODOS}

Foi realizado um estudo transversal com abordagem quantitativa com indivíduos acima de 60 anos e diagnosticados hipertensos, cadastrados em Unidades de Saúde da Família.

A seleção da amostra foi realizada após a consulta médica, onde todos os idosos diagnosticados hipertensos foram convidados a participar da pesquisa. Essa foi constituída pelos seguintes critérios de inclusão, idosos com idade $\geq 60$ anos, diagnosticado hipertensos (com uso ou não de medicação), não praticante de exercício físico, disponibilidade de realizar todas as atividades descritas na pesquisa e ser cadastrados nas Unidades de Saúde da Família (USF).

Como critérios de exclusão idosos institucionalizados (prisões, asilos, hospitais) com incapacidade mental severa, saúde debilitada ausência no dia, hora e local marcado para a avaliação. O estudo foi previamente aprovado pelo Comitê de Ética em Pesquisa do Centro de Ciências da Saúde da Universidade Federal de Pernambuco sob o no 2.754.151 de aprovação e CAAE № 89789918.9.0000.5208.

Foram realizados três encontros com os participantes, sendo no primeiro encontro todos foram informados sobre a pesquisa, assinaram o termo de consentimento livre e esclarecido (TCLE), foi aplicado o questionário utilizado pela Classificação Econômica Brasil (ABEP) para mensurar o nível socioeconômico, foi aplicado o questionário internacional de atividade física (IPAQ) para avaliar o nível de atividade física, realizamos a avaliação da pressão arterial, o índice de massa corporal (IMC), a circunferência do pescoço (CP) e a circunferência abdominal (CABS).

No segundo encontro todos receberam orientações através de exposições dialogadas e dinâmicas, realizada sobre hábitos saudáveis, consumo ideal de alimentos, informações sobre a realização da prática diária de atividade física com demonstrações de exercícios de flexibilidade, força muscular e cardiorrespiratório.

No terceiro e último encontro foram realizadas as mesmas atividades propostas do segundo com duração de 60 minutos, estes foram informados que deveriam realizar sessões de exercícios durante duas vezes por semana em casa e adequar seu comportamento diário com hábitos saudáveis durante dois meses e caso tenham dúvidas poderiam entrar em contato com o profissional responsável na Unidade de Saúde que eram atendidos para serem esclarecidos suas dúvidas, terminando os dois meses estes voltariam para serem avaliados novamente.

Para avaliar o nível socioeconômico foi utilizado o Critério de Classificação Econômica Brasil, organizado pela Associação Brasileira de Empresas de Pesquisa (ABEP). Esse questionário é composto por dois módulos de perguntas: o primeiro é em relação a bens materiais e domiciliar (por exemplo, quantidade de 
automóvel, geladeira, banheiros, etc.), e o segundo sobre o grau de instrução do chefe de família. A classificação é através do total dos pontos obtidos de acordo com as classes econômicas A1, A2, B1, B2 (alta), C1, C2 (média), D e E (baixa).

No nível de atividade física foi utilizada a versão curta do Questionário Internacional de Atividade física (IPAQ), composto por sete questões abertas onde suas informações permitem estimar o tempo semanal gasto em atividades físicas de intensidade moderada e vigorosa, em diferentes contextos do cotidiano, como: trabalho, transporte, tarefas domésticas e lazer, e ainda o tempo despendido em atividades passivas, realizadas na posição sentada. Através desse questionário os indivíduos hipertensos foram classificados como muito ativo, ativo, irregularmente ativo $A$ (realiza 10 minutos contínuos de atividade física conforme os critérios, frequência de 5 dias por semana ou duração de 150 minutos por semanas, irregularmente ativo $B$ (indivíduos que não atingem nenhum dos critérios citados no irregularmente ativo A) e sedentário (OLIVEIRA LMO, et al., 2018).

A pressão arterial (PA) foi avaliada utilizando um esfigmomanômetro e estetoscópio de marca Premium $\AA$, pelo método auscultatório, duas vezes, no braço esquerdo, após 10 minutos de repouso na posição sentada. A HA foi definida pelas médias pressóricas $\geq 140 / 90 \mathrm{mmHg}$ e/ou pelo uso de medicações (LAVIE CJ, et al., 2015).

O índice de massa corporal foi calculado pela relação entre a massa corporal $(\mathrm{kg})$ e o quadrado da estatura (m), utilizando uma balança digital de marca Hc022 Serene com capacidade de $180 \mathrm{~kg}$ e escala de $100 \mathrm{~g}$ e um estadiômetro portátil de marca Avanuti com $2 \mathrm{~m}$. Foi utilizada a classificação para idoso segundo a Organização Mundial de Saúde: baixo peso $<22 \mathrm{~kg} / \mathrm{m}^{2}$, peso adequado $\geq 22$ e $\leq 27 \mathrm{~kg} / \mathrm{m}^{2}$ e sobrepeso $>27$ $\mathrm{kg} / \mathrm{m}^{2}$.

A circunferência do Pescoço (CP) foi medida utilizando uma trena antropométrica de marca Prime Med com $1,50 \mathrm{~m}$, sendo medida na base do pescoço na altura da cartilagem cricotireoideana. Na presença de proeminência, em homens, a medida foi realizada abaixo da proeminência. Os valores de referência utilizados para a classificação da CP apresentando risco cardiovascular diminuído foram inferiores a $37 \mathrm{~cm}$ para homens e $34 \mathrm{~cm}$ para mulheres e, para risco cardiovascular aumentado, valores superiores a $37 \mathrm{~cm}$ para homens e superiores a $34 \mathrm{~cm}$ para mulheres (EKELUND U, et al., 2016).

A circunferência Abdominal (CABS) foi avaliada através de uma trena antropométrica de marca Prime Med com 1,50 m. Considerando a classificação da OMS, que estabelece como ponto de corte para risco cardiovascular aumentado medida de circunferência abdominal $\geq 94 \mathrm{~cm}$ em homens e $\geq 80 \mathrm{~cm}$ em mulheres (BRASIL, 2010).

Para a análise estatística utilizou-se os Softwares SPSS 20.0 (Statistical Packege for the Social Sciences) para Windows e o Excel versão 2013. Todos os testes foram aplicados com um intervalo de confiança de $95 \%$ e um nível de significância de $p<0,05$. Para verificar a normalidade utilizou-se o Teste de Normalidade de Shapiro-Wilk. Com finalidade de comparação antes e depois do mesmo grupo, utilizou-se o Teste t para amostras pareadas (Distribuição Normal) e Teste de Wilcoxon (Não Normal). Para comparação entre grupos e para efeito do tempo sobre os mesmos, utilizou-se ANOVA mista com variáveis dependentes.

\section{RESULTADOS}

O cálculo amostral foi realizado com 120 idosos, admitindo-se um erro amostral de 5\% com um nível de confiança de $95 \%$, finalizando um total de 49 idosos, sendo excluídos 50 por não preencherem os critérios de inclusão e 21 por não voltarem e ou se recusarem a fazer parte do projeto pós-intervenção, todos com faixa etária de 60 a 80 anos com média de $67,92( \pm 6,24),(71,0 \%)$ do sexo feminino e $(28,5 \%)$ do sexo masculino, (36,7\%) viúvos, $(89,7 \%)$ tem ensino fundamental incompleto, e renda mensal de um a três salários mínimos $(51,0 \%)$. As medicações mais usadas pelos participantes para o controle da HA foram: losartana e hidroclorotiazida. 
Tabela 1 - Caracterização dos participantes da pesquisa de acordo com número de pessoas e o percentual para cada variável $(n=49)$.

\begin{tabular}{lc}
\hline Variáveis & $\mathbf{n}(\%)$ \\
\hline Sexo & $14(28,5)$ \\
\hline Masculino & $35(71,4)$ \\
\hline Feminino & \\
\hline Estado civil & $13(26,5)$ \\
\hline Solteiro & $15(30,6)$ \\
Casado & $3(6,1)$ \\
Divorciado & $18(36,7)$ \\
Viúvo & \\
\hline Escolaridade & $44(89,7)$ \\
\hline Ensino básico incompleto & $4(4,1)$ \\
Médio completo & \\
\hline Renda mensal & $24(48,9)$ \\
\hline Até 1 salário mínimo & $25(51,0)$ \\
1 a 3 salários mínimos & $33(67,3)$ \\
\hline Medicação para hipertensão & $24(48,9)$ \\
\hline Losartana & \\
Hidroclorotiazida & \\
\hline
\end{tabular}

Fonte: Silva GSM, et al., 2021.

Em relação ao critério de classificação econômica, os participantes da pesquisa são da classe D-E (26 pessoas), sendo considerada a mais baixa de acordo com o critério socioeconômico, seguida pelas demais, sendo mostrados na Figura 1.

Figura 1 - Critério de classificação econômica pelo questionário da Associação Brasileira de Empresas de Pesquisa (ABEP) na amostra $(n=49)$.

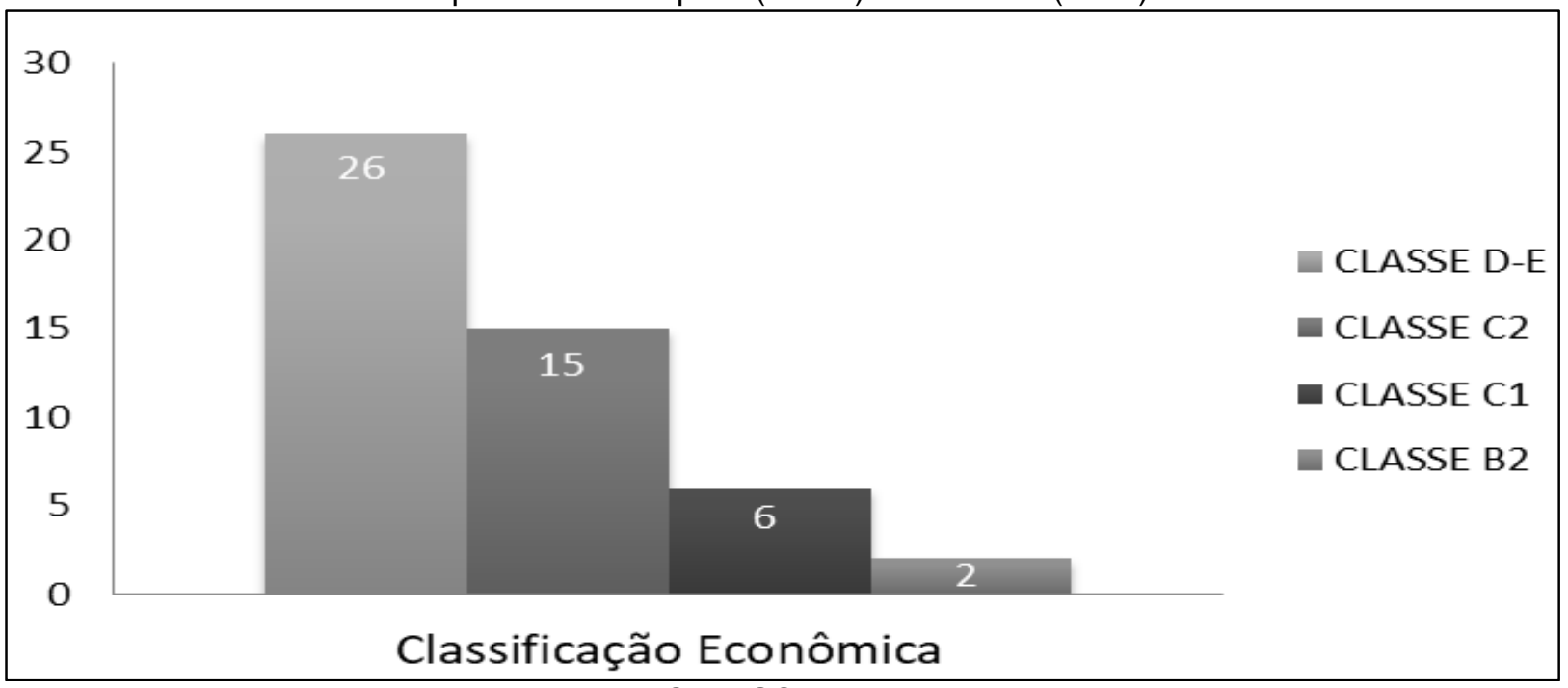

Fonte: Silva GSM, et al., 2021

O nível de atividade física foi avaliado pelo IPAQ, na Figura 2, os resultados mostram que houve uma melhora significante $(\mathrm{p}=0,014)$ do nível de atividade física dos idosos na segunda avaliação (2a) quando comparada com a primeira $\left(1^{\stackrel{a}{a})}\right.$, o número de idosos ativos aumentou e o de sedentários diminuiu. 
Figura 2 - Classificação do nível de atividade física da primeira e segunda avaliação dos participantes, através do Questionário Internacional de Atividade Física - IPAQ ( $n=49)$.

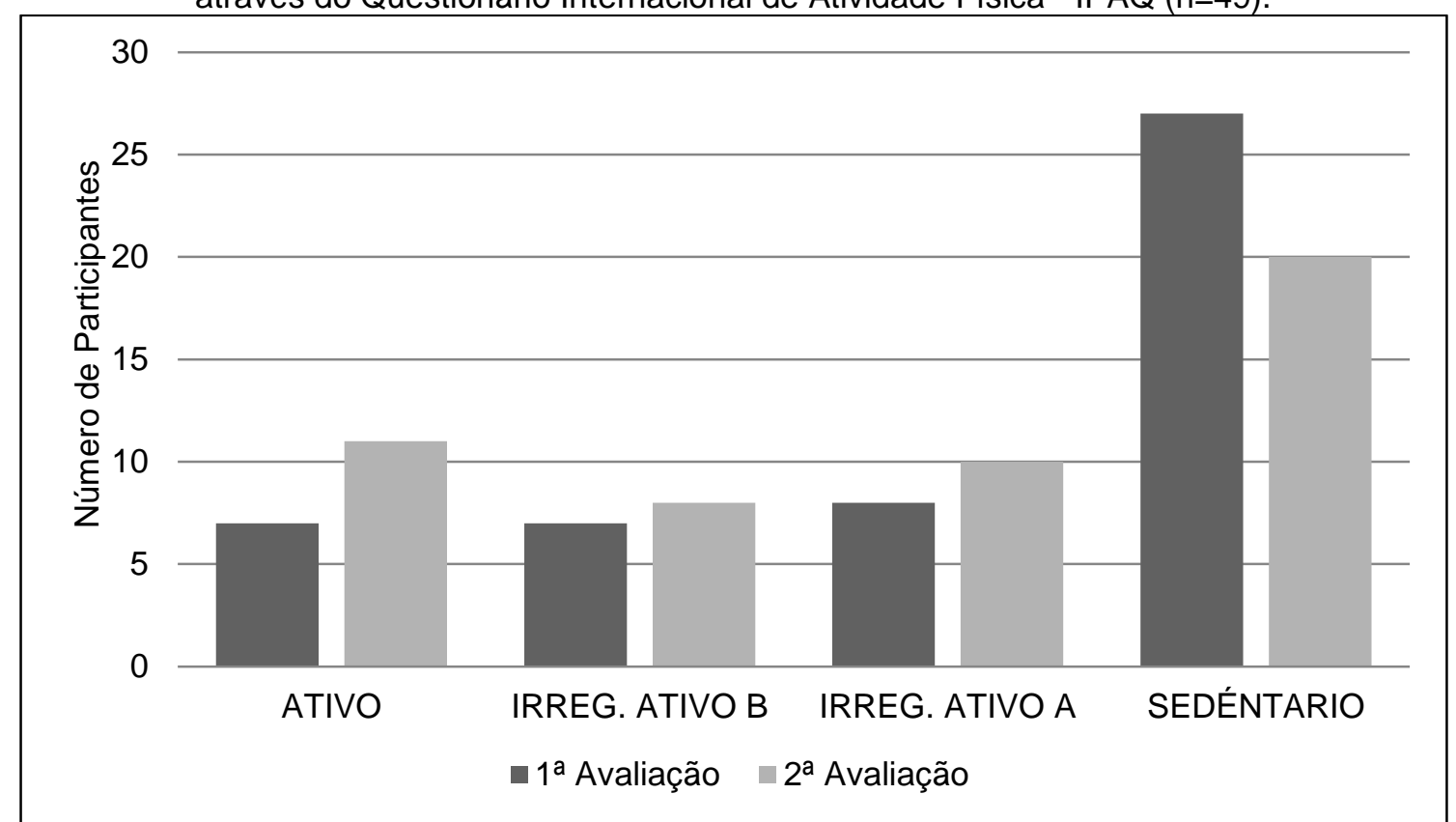

Legenda: IRREG= irregularmente. $p=0,014$ (O valor de $p$ se refere ao Teste t de amostra em pares).

Fonte: Silva GSM, et al., 2021.

$\mathrm{Na}$ Tabela 2 os resultados mostram que houve uma melhora significativa na pressão arterial sistólica, não havendo diferença na diastólica. Em relação às medidas antropométricas, houve diferença significativa em todas as variáveis na segunda avaliação quando comparadas com a primeira.

Tabela 2 - Diferença da pressão arterial e medidas antropométricas entre a primeira e a segunda avaliação.

\begin{tabular}{cccc}
\hline Variáveis & 1 avaliação & 2o avaliação & Valor de $\boldsymbol{p}$ \\
\hline PAS & $137,14 \pm 22,27$ & $126,73 \pm 16,88$ & 0,001 \\
PAD & $83,06 \pm 11,76$ & $80,27 \pm 8,15$ & 0,084 \\
PESO & $71,99 \pm 13,80$ & $71,27 \pm 13,43$ & 0,002 \\
IMC & $28,40 \pm 4,64$ & $28,16 \pm 4,39$ & 0,015 \\
CP & $36,16 \pm 3,51$ & $35,71 \pm 3,34$ & 0,001 \\
CABS & $98,35 \pm 11,62$ & $97,59 \pm 11,16$ & 0,007
\end{tabular}

Legenda: $P A S=$ Pressão Arterial Sistólica; $P A D=$ Pressão Arterial Diastólica; $I M C=$ Índice de Massa Corporal; $\mathrm{CP}=$ Circunferência do Pescoço; $\mathrm{CABS}=$ Circunferência Abdominal. $\mathrm{O}$ valor de $\mathrm{p}$ se refere ao teste $t$ de amostras em pares.

Fonte: Silva GSM, et al., 2021.

A Tabela 3, mostra a classificação do risco cardiovascular segundo as medidas antropométricas e classificação do IMC, a correlação e t da circunferência abdominal não podem ser calculados porque o erro padrão da diferença é 0 . Em relação ao $\mathrm{CP},(55,1 \%)$ das pessoas estavam com risco cardiovascular aumentado na primeira avaliação, diminuindo esse valor para $(48,9 \%)$ na segunda avaliação. A CABS mostrou que $(83,6 \%)$ dos idosos apresentaram rico cardiovascular, não havendo nenhuma diferença de classificação em relação à segunda avaliação. $\mathrm{Na}$ avaliação do $\mathrm{IMC}$, os idosos apresentaram na primeira um aumento de sobrepeso e um pequeno número de peso adequado, porém na segunda estes tiveram uma diminuição e aumento respectivamente, sendo verificado que o baixo peso se manteve igual. 
Tabela 3 - Classificação do risco cardiovascular de acordo com medidas antropométricas e classificação do IMC, através do número de pessoas e percentual por variável $(n=49)$.

\begin{tabular}{lccc}
\hline Variáveis & $\begin{array}{c}\mathbf{1}^{\mathbf{a}} \text { avaliação } \\
\mathbf{n}(\%)\end{array}$ & $\begin{array}{c}\mathbf{2}^{\mathbf{a}} \text { avaliação } \\
\mathbf{n}(\%)\end{array}$ & $\boldsymbol{p}$ \\
\hline Circunferência do pescoço & & & 0,083 \\
\hline Sem risco & $22(44,8)$ & $25(51,0)$ & \\
Risco aumentado & $27(55,1)$ & $24(48,9)$ & 0,007 \\
\hline Circunferência abdominal & & & \\
\hline Sem risco & $8(16,3)$ & $8(16,3)$ & 0,159 \\
Risco aumentado & $41(83,6)$ & $41(83,6)$ & \\
\hline Classificação do IMC & & & \\
\hline Peso adequado & $18(36,7)$ & $20(40,8)$ & \\
Sobrepeso & $29(59,1)$ & $27(55,1)$ & \\
Baixo peso & $2(4,0)$ & $2(4,0)$ & \\
\hline
\end{tabular}

Legenda: $O$ valor do $p$ se refere ao Teste t de amostra em pares.

Fonte: Silva GSM, et al., 2021.

\section{DISCUSSÃO}

Através dos dados obtidos no presente estudo foi possível verificar que a intervenção baseada na promoção e educação na saúde com realização de exercícios físicos, nível de atividade física, pressão arterial, índice de massa corporal (IMC), circunferência do pescoço e circunferência abdominal foram eficazes para estimular os idosos participantes da pesquisa a desenvolverem um novo padrão diário de comportamento.

Sobre o nível de atividade física, na primeira avaliação uma grande parte dos idosos não praticavam atividade física ou estavam a abaixo do nível recomendado. Tais resultados foram encontrados no estudo de Benedetti TRB, et al. (2007), onde observou que a maioria dos idosos $(66,10 \%)$ da sua pesquisa foram classificados com níveis insuficientes de atividade física avaliado pelo IPAQ.

Nossos resultados encontrados neste estudo mostram que houve uma redução nas medidas da circunferência do pescoço, IMC e circunferência abdominal quando comparados com o início da intervenção de Ekelund U, et al. (2016). Estes resultados apontam a importância de intervenções para idosos na atenção primaria. Ekelund U, et al. (2016) e Ministério da Saúde (BRASIL, 2010) ambos indicam a busca de novos comportamentos e inserção da prática de atividades físicas diárias em grupos de idosos hipertensos

Outros dados foram encontrados numa pesquisa internacional utilizando o mesmo questionário, mostrando diferença no volume da atividade física no IMC, visto que o volume de atividade física nos idosos com sobrepeso e obesidade é muito baixo, também verificado em todos os domínios de atividades físicas analisadas (relacionadas ao trabalho, transporte, tarefas domésticas e de lazer), quando comparados com idosos com peso normal (SBC, 2016).

Especialmente nos idosos, o sedentarismo está relacionado de forma negativa à saúde e quando comparados com outras idades, são considerados os que mais passam tempo em comportamento sedentário, aumentando o risco de mortalidade nessa faixa etária. Diante disso, a literatura apresenta alguns fatores que contribuem para a inatividade física nesse público, como por exemplo, o baixo nível socioeconômico, comprometimento funcional, presença de doenças, medo de ocorrência de lesão, falta de companhia, local e clima inadequado (FRIZON V e BOSCAINI C, 2013; ABESO, 2009).

A pressão arterial apresentou-se alterada na maioria dos idosos quando comparada com a primeira avaliação, mostrando que os comportamentos destes tiveram uma mudança, devido à reeducação de seus hábitos. As medidas antropométricas mostraram o excesso de peso, aumento da circunferência do pescoço e do abdome, além da classificação de sobrepeso de acordo com o IMC em grande parte dos idosos, 
representando um alto risco cardiovascular. Esses resultados foram prevalentes durante as duas avaliações, mesmo tendo uma redução entre as medidas de circunferência do pescoço e do IMC na segunda avaliação, os índices de sobrepeso e o risco cardiovascular aumentado continuaram altos (LEVORATO CD, et al., 2014).

Os dados dessa pesquisa apresentam uma associação com outros estudos nacionais, que têm demonstrado aumento prevalente de excesso de peso na população idosa e a literatura também tem mostrado que idosos com idades menores apresentam valores mais elevados de IMC em comparação com os que têm mais de 70 anos. O excesso de peso está relacionado com aumento da prevalência da HA, em todas as idades. No entanto, perder peso e reduzir a circunferência abdominal é um fator importante para a diminuição da pressão arterial, melhorando também as alterações metabólicas relacionadas a esse problema. Onde podemos identificar que em nossos achados estes resultados foram relevantes para esta diminuição (CIPRIANO AS, et al., 2018; COSTA FR, et al., 2018; FLORES TR, et al., 2018; GALDIANO AM, et al., 2018; ZBRONSKA I e MEDRELA-KUDER E, 2018).

Os resultados desse estudo confirmam os dados encontrados por Silveira, et al. (2018), onde 50\% da amostra de idosos foram acometidos com aumento prevalente da circunferência abdominal. Bombardelli, et al. (2017), investigou os fatores de risco cardiovascular em idosos usuários do SUS de Goiânia e observou que houve prevalência de aumento da circunferência da cintura em 83,3\% dos 418 participantes do estudo, sendo considerados acima do padrão ideal.

Nosso estudo apresenta resultado semelhante ao realizado por Corrêa MM, et al. (2017), onde idosos institucionalizados foram classificados como um grupo vulnerável para fatores de risco cardiovascular, pois as medidas antropométricas apresentaram-se com valores além do normal, onde 47,40\% das mulheres e $36,12 \%$ dos homens estavam com sobrepeso e $76,16 \%$ das mulheres e $41,06 \%$ dos homens com a circunferência da cintura acima do recomendado. Além disso, dados de um estudo que avaliou a circunferência do pescoço com o IMC na ocorrência de excesso de peso em idosos mostrou que 74,2\% dos avaliados apresentaram circunferência do pescoço aumentada, concordando com os resultados da atual pesquisa (VAGETTI GC, et al., 2017).

De acordo com o Critério de classificação econômica a maioria dos participantes da pesquisa pertence ao nível econômico D-E, sendo este considerado o nível mais baixo da classificação para o país, levando em consideração o fato de que a pesquisa foi realizada em bairros vulneráveis do Recife, tendo em vista a localização das USF.

Todos os participantes do presente estudo foram orientados e receberam um manual ilustrativo durante a segunda e terceiro encontro, contendo informações sobre a importância e como praticar atividade física, as ações de controle da pressão arterial, além de conscientização sobre práticas de estilo de vida que proporcionam melhores condições de saúde, como por exemplo, a alimentação saudável e o controle do peso corporal.

No último encontro de avaliação foi observado que houve melhora nos níveis de atividade física entre os idosos, após as orientações, além das medidas antropométricas como foi descrito anteriormente, isso reflete positivamente sobre a prática da orientação que os profissionais de saúde da atenção básica podem realizar para que os pacientes tenham um cuidado mais relevante com a saúde.

Outros achados também sugerem que receber orientações pode contribuir positivamente para a redução do consumo de alimentos inadequados, além de diminuir o excesso de sal e aumentar a prática de atividade física em idosos (SUSIC D e VERAGIC J, 2017).

Mesmo o Ministério da Saúde elaborando políticas de promoção e educação em saúde, ainda é possível perceber que essas orientações não são muito realizadas no contexto dos serviços de atenção básica (POOROLAJAL J, et al., 2016).

O aconselhamento de profissionais de saúde sobre hábitos saudáveis é muito importante, assim como a realização efetiva dessas recomendações em nível individual. Essa é uma prática educativa em que 
profissionais do âmbito da atenção básica têm como alvo tornar os indivíduos atentos sobre seu processo de saúde, respeitando à autonomia e valorizando seu potencial, levando as alterações comportamentais e melhoras na qualidade de vida das pessoas (SILVEIR EA, et al., 2018; SCIANNI AA, et al., 2019).

É necessário que a conscientização seja contínua para que a população entenda cada vez mais a importância de manter um estilo de vida saudável, e dessa forma, aumentar as possibilidades de ampliar o acesso à saúde e a atenção básica, garantindo a educação popular em saúde, incentivando as práticas saudáveis e evitando dessa forma a prevalência de sobrepeso, obesidade, diabetes e hipertensão (FLORES TR, et al., 2018; REIS GMS, et al., 2018).

Observamos que este estudo apresentou algumas limitações, como a natureza da pesquisa ser transversal e o pequeno número da amostra, que apesar de ter sido um total de todos os idosos diagnosticados hipertensos nas duas USF estudadas, esta coleta foi desenvolvida apenas nas unidades cadastradas no programa de residência multiprofissional da saúde da família da UFPE - Centro de Ciências da Saúde. Além disso hoje o número de pesquisas sobre o tema é escasso e por não ter uma amostra representativa, onde os resultados não podem ser estendidos para a toda a população de idosos hipertensos da população brasileira.

A pesquisa demonstrou uma melhora nos níveis de atividade física, na pressão arterial e entre os aspectos antropométricos como IMC e circunferência do pescoço nos idosos hipertensos, após a conscientização sobre hábitos saudáveis do estilo de vida e promoção da saúde.

\section{CONCLUSÃO}

Concluímos que a criação de programas de intervenção de Educação em Saúde no SUS mostrou uma melhora e mudança no comportamento do usuário sobre seu estilo de vida e promoção da saúde, apresentado em nosso estudo como melhoria nos níveis de atividade física, pressão arterial, redução do IMC, circunferência do pescoço e circunferência abdominal criando assim na população de idosos hipertensos uma melhoria e manutenção na sua qualidade de vida.

\section{REFERÊNCIAS}

1. ABESO. Associação Brasileira para o Estudo da Obesidade e da Síndrome Metabólica. Diretrizes brasileiras de obesidade. São Paulo, 2009.

2. BENEDETTI TRB, et al. Reprodutibilidade e validade do Questionário Internacional de Atividade Física (IPAQ) em homens idosos. Rev Bras Med Esporte. 2007; 13(1): 11-16.

3. BOMBARDELLI C, et al. Quality of life elderly people living in a municipality with rural characteristics in the countryside of Rio Grande do Sul. Rev Bras Geriat e Gerontol. 2017; 20(1): 88-94.

4. BRASIL. Ministério da Saúde. Departamento de Vigilância de Doenças e Agravos não Transmissíveis e Promoção da Saúde. Vigitel 2016: vigilância de fatores de risco e proteção para doenças crônicas por inquérito telefônico: estimativas sobre frequência e distribuição sociodemográfica de fatores de risco e proteção para doenças crônicas nas capitais dos 26 estados brasileiros e no Distrito Federal em 2016/ Secretaria de Vigilância em Saúde e Promoção da Saúde: Disponível em: https://portalarquivos2.saude.gov.br/images/pdf/2018/marco/02/vigitel-brasil2016.pdf.

5. CIPRIANO AS, et al. A influência do Exercício Físico Resistido na qualidade de vida do idoso. Revista Eletrônica Acervo Saúde. 2011; 2: 70-84.

6. COSTA FR, et al. Qualidade de vida de idosos participantes e não participantes de programas públicos de exercícios físico. Rev Bras Geriatr e Gerontol. Rio de Janeiro. 2018; 27(1): 24-34.

7. CORREAA MM, et al. Razão cintura-estatura como marcador antropométrico de excesso de peso em idosos brasileiros. Cad Saúde Pública. 2017; 33(5): 1678-4464

8. DAMMERO DR, et al. Perfil e estado nutricional de pacientes hipertensos atendidos em um ambulatório de nutrição do sul do Brasil. Revista Brasileira de Obesidade, Nutrição e Emagrecimento, São Paulo. 2019; V. 13 (77): $54-60$.

9. FARIAS NO, et al. Aspectos cognitivos da pessoa idosa na aposentadoria: uma revisão sistemática. Revista Eletrônica Acervo Saúde, 2021, 13(2): e5957.

10. EKELUND U, et al. Does physical activity attenuate, or even eliminate, the detrimental association of sitting time with mortality? A harmonised meta-analysis of data from more than 1 million men and women. The Lancet. 2016; 388(10051): 1302-1310.

11. FERREIRA RC, et al. Perfil clínico-epidemiológico dos portadores de hipertensão atendidos na atenção básica do estado de Alagoas. Medicina (Ribeirão Preto, Online). 2017; 50(6): 349-357. 
12. FLORES TR, et al. Aconselhamento por profissionais de saúde e comportamentos saudáveis entre idosos: estudo de base populacional em Pelotas, sul do Brasil, Epidemiol. Serv. Saúde. 2018; 27(1): 15.

13. FRIZON V, BOSCAINI C. Circunferência do pescoço, fatores de risco para doenças cardiovasculares e consumo alimentar. Rev Bras Cardiol. 2013; 26(6): 426-34.

14. GALDIANO AM, et al. Associação entre nível de atividade física, indicadores de saúde e qualidade de vida de idosos da universidade da maturidade, Palmas-TO. Rev Cereus. 2018; 10(3): 165-178.

15. CASTRO L, et al. Perfil epidemiológico da hipertensão arterial sistêmica em uma população da zona urbana do Maranhão. Revista Eletrônica Acervo Saúde, 2019; (18): 125.

16. HALL JE, et al. Obesity-induced hypertension: interaction of neurohumoral and renal mechanisms. Circ Res, 2015; 116(6): 991-1006.

17. HERROD PJJ, et al. Exercise and other non-pharmacological strategies to reduce blood pressure in older adults: a systematic review and meta-analysis. Journal of the American Society of Hypertension, 2018; 1-60.

18. LEANDRO MPG, et al. Efeito do componente aeróbio no treinamento combinado sobre a pressão arterial de idosas hipertensas. Revista Brasileira de Medicina do Esporte, 2019; 25: 469-473.

19. LAVIE CJ, et al. Exercise and the cardiovascular system: clinical science and cardiovascular outcomes. Circ Res. 2015; 117(2): 207-19.

20. LEVORATO CD, et al. Fatores associados à procura por serviços de saúde numa perspectiva relacional de gênero. Ciênc. saúde coletiva, 2014; 19(4): 1263-1274.

21. MIRANDA GMD, et al. O envelhecimento populacional brasileiro: desafios e consequências sociais atuais e futuras. Rev Bras Geriat Gerontol. 2016; 19(3): 507-519.

22. MIURA H, et al. Effects of exercise training on arterial stiffness in older hypertensive females. Eur J Appl Physiol. 2015; 115: 1847-1854.

23. MOZAFFARIAN D, et al. American Heart Association Statistics Committee and Stroke Statistics Subcommittee. Heart disease and stroke statistics-2015 update: A report from the American Heart Association. Circulation. 2015 ; 131 (4): e 29-322.

24. NASCHITZ JE. Blood pressure management in older people: balancing the risks. Postgrad Med $\mathrm{J}$ Epub ahead of print; 2018; 94(1112):348-353.

25. OLIVEIRA LMO, at al. Resposta de pacientes hipertensos sob tratamento medicamentoso de acordo com os níveis pressóricos. Acta Biomedica Brasiliensia. 2018; 9(3): 2236-0867.

26. OLIVEIRA VS, et al. Análise dos fatores de risco para doenças crônicas não transmissíveis: estudo com colaboradores de uma instituição privada. Santa Maria. 2017; 43(1): 214-224.

27. POOROLAJAL J, et al. How much excess weight loss can reduce the risk of hypertension. J Public Health. 2016; 39(3): 95-102.

28. PORTES LA, et al. Avaliação do estado nutricional e composição corporal de idosos de Embú-Guaçu-SP. RBONERevista Brasileira de Obesidade, Nutrição e Emagrecimento, 2018; 12(72): 446-455.

29. RAMOS RF, et al. Efeitos benéficos da dança na saúde do idoso. Revista Eletrônica Acervo Saúde. 2018 ; (2): 77-85.

30. REIS GMS, et al. Circunferência do pescoço como indicador de excesso de peso em idosas. Rev Bras Obes, Nutr e Emagrecimento. 2018; 12 (75 Suppl1): 942-947.

31. SCIANNI AA, et al. Efeitos do exercício físico no sistema nervoso do indivíduo idoso e suas consequências funcionais. Resv. Bras Ciên Esporte. 2019; 41(1): 81-95

32. SILVEIRA EA, et al. Elevada prevalência de obesidade abdominal em idosos e associação com diabetes, hipertensão e doenças respiratórias. Ciênc \& Saúde Colet. 2018; 23(3): 903-912.

33. Sociedade Brasileira de Cardiologia (SBC). 7ª Diretriz Brasileira de Hipertensão Arterial. Arq Bras Cardiol. 2016; 107(Supl.3): 1-83.

34. SUSIC D, VARAGIC J. Obesity: a perspective from hypertension. Med Clin North Am. 2017; 101(1): $139-57$.

35. VAGETTI GC, et al. Associação do índice de massa corporal com a aptidão funcional de idosas participantes de um programa de atividade física. Rev Bras Geriat e Gerontol. 2017; 20(2): 216-227.

36. World Health Organization. Global status report on noncommunicable diseases 2010. Description of the global burden of NCDs, their risk factors and determinants. Geneva: World Health Organization; 2010.

37. ZBROŃSKA I, MĘDRELA-KUDER E. The level of physical activity in elderly persons with overweight and obesity. Rocz Panstw Zakl Hig. 2018; 69(4): 369-373. 